\title{
Spot urine pancreolauryl test for use in infancy
}

\author{
M R Green, S Austin, P McClean, S Jolliffe, L T Weaver
}

\begin{abstract}
As a simple non-invasive test of possible pancreatic insufficiency 10 healthy infants, 13 infants with cystic fibrosis, and nine infants with unexplained diarrhoea and failure to thrive were given an emulsion containing fluorescein dilaurate and mannitol by mouth. A spot urine specimen was collected and results expressed as urinary fluorescein to mannitol ratios. Sensitivity of the test was $96 \%$ and specificity was $95 \%$.

(Arch Dis Child 1995; 72: 233-234)
\end{abstract}

Keywords: exocrine pancreatic function, non-invasive tests, pancreolauryl test.

Measurement of pancreatic exocrine function in infancy presents many problems. We previously described a modified pancreolauryl test which eliminates the need for a two day urine collection. ${ }^{1}$ We describe further modifications of the test to make it suitable for repeated use in infancy. The currently available pancreolauryl test requires ingestion of hard gelatin capsules. To use the test in infants a sterile unit dose emulsion was developed so that the required ratio of fluorescein dilaurate and mannitol could be added to an infant feed either immediately before ingestion or given in liquid form.

After fluorescein dilaurate and mannitol are ingested, fluorescein is cleaved from fluorescein dilaurate by pancreatic aryl esterases and free fluorescein is absorbed and excreted in the urine where it can be measured spectrophotometrically. ${ }^{2}$ Mannitol resists hydrolysis in the stomach and small intestine, is passively absorbed, and is excreted in urine ${ }^{3}$ where it can be measured enzymatically. ${ }^{4}$ Fluorescein recovery in the urine is therefore an indirect measure of pancreatic esterase activity and absorption, and mannitol recovery of intestinal uptake. The test result is expressed as a ratio of free fluorescein recovery to mannitol recovery.

MRC Dunn Nutrition Unit, Cambridge M R Green $S$ Austin

P McClean

L T Weaver

Charwell Health Care, Alton, Hampshire S Jolliffe

Correspondence to: Dr M R Green, The Children's Hospital, Leicester Royal Infirmary, Leicester LE1 5WW.

Accepted 30 November 1994

\section{Subjects and methods}

SUBJECTS

Ten healthy infants (five boys, five girls) aged 5-17 months (median $12 \cdot 5$ months) acted as controls. Two children were studied twice. Thirteen infants with cystic fibrosis (six boys, seven girls) aged 2-76 months (median 14 months) were studied on a total of 27 occasions. The diagnosis of cystic fibrosis was confirmed by sweat test and 12 infants were judged clinically to require pancreatic enzyme supplementation. Ten children were studied twice and two children on three occasions.
Nine infants with unexplained diarrhoea and failure to thrive (six boys, three girls) aged 2-42 months (median 16 months) were studied each on one occasion. Two children had steatorrhoea, two children chronic diarrhoea, one had juvenile chronic arthritis with failure to thrive, and four children had unexplained failure to thrive with weights below the third centile.

\section{TEST PROCEDURE}

The studies were made in the morning after an overnight fast unless the infants were still receiving regular milk feeds in which case the fast did not exceed four hours. Fluorescein dilaurate $(0.134 \mathrm{mmol})$ and mannitol $(6 \cdot 7$ $\mathrm{mmol}$ ) in $10 \mathrm{ml}$ of emulsion were given by mouth followed by a milk feed. The emulsion was formed of the water insoluble fluorescein dilaurate ester dissolved in a medium chain triglyceride, using a soya bean based lecithin as emulsifier. Ultrasonication or microfluidisation produced an extremely fine dispersion of the triglyceride in the aqueous phase, which contained the second marker, mannitol. The emulsion was physically stable, resistant to heat sterilisation, and readily dispersible in infant milk formulas by simple shaking.

Feeds were given thereafter as desired with or without pancreatic supplements as required. The two 5 year old children studied were allowed breakfast of tea, toast, and cereal two hours after taking the test substrates. Specimens of urine were collected as voided, into a urine bag, or using cotton wool balls enclosed in the napkins in infants, ${ }^{5}$ including in each study a specimen produced after two, but before five, hours after substrate ingestion. Urine was frozen at $-20^{\circ} \mathrm{C}$ until analysis.

BIOCHEMICAL MEASUREMENTS

Fluorescein concentration was measured spectrophotometrically. An aliquot of $0.5 \mathrm{ml}$ urine was added to $4.5 \mathrm{ml} 0.1 \mathrm{M}$ sodium hydroxide and incubated at $70^{\circ} \mathrm{C}$ for 10 minutes to hydrolyse any fluorescein glucuronide (which is colourless) to free fluorescein. After centrifugation the absorbance (A) of the supernatant was measured at $492 \mathrm{~nm}$ against water. Fluorescein concentration was calculated from the equation:

$$
\left(\left(\left(A_{492} \times 100\right) / 35\right) \times(50 \cdot 42 / 100)\right) \times 50
$$

The denominator and multiplication factors were determined from the dose administered, the dilution, and the absorption coefficient. Mannitol concentration was measured enzymatically using a method previously described. ${ }^{1} 4$ The test result was expressed as a ratio of 


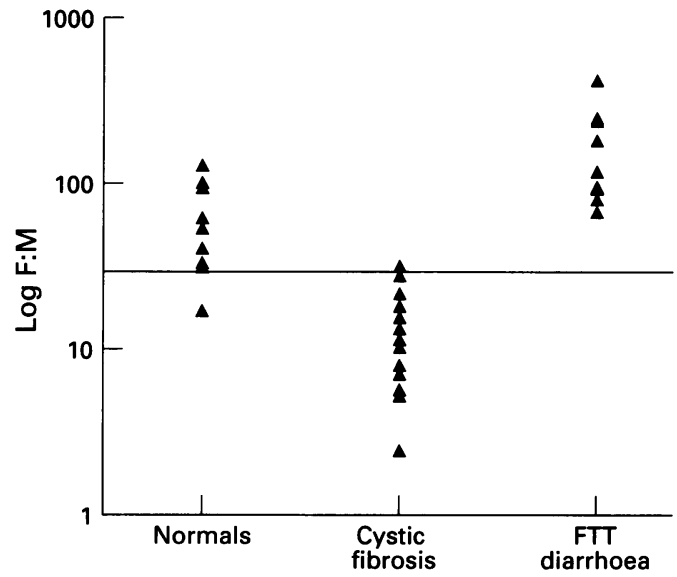

Log transformed $F: M$ concentration ratios measured in urine specimens collected between two and five hours after substrate ingestion. The line indicates 30 , which is the cut off point between normal and abnormal exocrine pancreatic function. For children who had more than one test performed the mean result is plotted. FTT=failure to thrive.

fluorescein concentration to mannitol concentration ( $\mathrm{F}: \mathrm{M}$ ratio).

The significance of the differences in $F: M$ ratios between the three groups was measured using the Mann-Whitney test. All studies were carried out with informed consent of parents of the children and approval of the local ethical committee.

\section{Results}

The median test $\mathrm{F}: \mathrm{M}$ ratio was $\mathbf{5 8 . 3}$ for normal subjects, 120 for infants with diarrhoea and failure to thrive, and 11.3 for children with cystic fibrosis. Because of the skewed nature of the data (high normal values in some children with failure to thrive and diarrhoea) the figure shows the $\log$ transformed $F: M$ ratio of a single urine specimen collected between two and five hours after substrate ingestion for each test.

The F:M ratios of the children with cystic fibrosis were significantly $(p<0.0001)$ lower than those of the normal children, and children with diarrhoea and failure to thrive. The test sensitivity was $96 \%$ and specificity $95 \%$.

\section{Discussion}

Using a combination of fluorescein dilaurate and mannitol, suspended in an emulsion, we have distinguished between infants and children with cystic fibrosis, and normal infants and those with diarrhoea and failure to thrive. We have previously described the rationale of the use of a dual marker system in the non-invasive assessment of exocrine pancreatic function and established the suitability of mannitol as the reference marker to fluorescein dilaurate. ${ }^{1}$ When a combination of fluorescein dilaurate and mannitol was given by mouth and the result expressed as a fluorescein to mannitol concentration ratio obtained from a spot specimen of urine collected at any time point between two and five hours after substrate ingestion, there was discrimination between children with abnormal exocrine pancreatic function and normal children and those with diarrhoea and failure to thrive. The results are not affected by the concurrent administration of pancreatic enzymes. ${ }^{1}$

The higher range of $F: M$ ratios of the children with diarrhoea and failure to thrive was largely due to diminished mannitol uptake and excretion, which is a well documented feature of small intestinal enteropathies. ${ }^{6}$ Our results do not suggest that the previously accepted cut off between normals and subjects with abnormal pancreatic function should be changed. A $F: M$ ratio of $<30$ represents pancreatic insufficiency and an $F: M$ ratio of $>30$ represents pancreatic sufficiency. This figure is based on a number of studies evaluating the pancreolauryl test including our own modification. ${ }^{1}$ In this study it is adopted again as an arbitrary line giving the best separation between disease and control subjects. Tests with ratios of between 20 and 30 should either be repeated or alternative measures of exocrine pancreatic function used. Because the test specifically measures the capacity of pancreatic aryl esterases to cleave fluorescein from lauric acid it will not detect some isolated enzyme deficiencies.

This new test was well tolerated by infants. In contrast with the two day pancreolauryl test a standardised breakfast was not necessary, nor was there a need to encourage a large fluid intake. Both factors are of importance if the test is to be used in infancy. The modified fluorescein dilaurate-mannitol test provides a simple, reliable, and tubeless test of exocrine pancreatic function. It eliminates the need for timed urine collections and a two day test and is eminently suitable for repeated use as a diagnostic test to distinguish infants and children with pancreatic insufficiency, from normal children, and those with other causes of diarrhoea and failure to thrive.

We thank the children, parents, and paediatricians who cared for them, and Charwell Health Care for their support. MRG was a CF Trust research fellow.

1 Green MR, Austin S, Weaver LT. Dual marker one day pancreolauryl test. Arch Dis Child 1993; 68: 649-52.

2 Meyer-Bertenrath JG. Lipolytic enzymes of the human pancreas. A highly sensitive, specific and simple measurement of cholesterol ester hydrolase. Enzyme 1982; 28: 336-42.

3 Laker MF, Bull HJ, Menzies IS. Evaluation of mannitol as a probe marker of gastrointestinal permeability in man. Eur $\mathcal{F}$ Clin Invest 1982; 12: 485-91.

4 Lunn PG, Northrop CA, Northrop AJ. Automated enzyme assays for the determination of intestinal permeability probes in urine. 2. Mannitol. Clin Chim Acta 1989; 183: probes in $163-70$.

5 Roberts SB, Lucas A. Measurement of urinary constituents and output using disposable napkins. Arch Dis Child 1985; and output

6 Weaver LT, Chapman PD, Madeley CR, Laker MF, Nelson RN. Intestinal permeability changes and excretion of micro-organisms in stools of infants with diarrhoea and vomiting. Arch Dis Child 1985; 60: 326-32. 